\title{
Lösungsmöglichkeiten bei fehlgeschlagenen Osteosynthesen - Humerusschaft
}

J. Christoph Katthagen, Philipp A. Michel, Benedikt Schliemann, Michael J. Raschke

\section{Epidemiologische Daten}

Humerusschaftfrakturen sind im Vergleich zu anderen Verletzungen eher selten und machen nur ca. 1-3\% aller Frakturen aus [1]. In der Literatur sind länderspezifische Inzidenzraten von 7-19 pro 100000 Einwohner beschrieben [2-4]. In den Industrienationen ist eine bimodale Altersverteilung mit einem Peak bei jungen männlichen Erwachsenen (Hochrasanztrauma beim Sport oder Verkehrsunfall) sowie älteren Frauen (Niedrigenergietrauma bei Osteoporose) typisch [5]. Am gesamten Humerus sind je nach Studie ca. 13-25\% echte diaphysäre Schaftfrakturen, während $47-79 \%$ den proximalen und $8-33 \%$ den distalen Humerus betreffen [6-8]. Nach Meinung einiger Autoren stellt die proximale Schaftfraktur als „intermuskuläre Fraktur" zwischen den humeralen Ansätzen der Mm. pectoralis, deltoideus und latissimus dorsi eine eigene Frakturentität dar und sollte nicht zu den Schaftfrakturen gezählt werden $[9,10]$. Als weitere Sonderform gilt die Holstein-Lewis-Fraktur, die als distale Spiralfraktur mit Einklemmung des N. radialis im Frakturspalt und entsprechenden neurologischen Ausfallerscheinungen definiert ist [11].

\section{Therapeutische Prinzipien am Humerusschaft}

\section{Stellenwert der konservativen Verfahren}

Die konservative Therapie hat, u.a. geprägt durch die Arbeiten von Böhler und Sarmiento, immer noch einen hohen Stellenwert in der Behandlung von Humerusschaftfrakturen. In einer Serie mit 620 Patienten konnten, durch Ruhigstellung des Oberarms in einem entsprechenden Brace (n. Sarmiento), gute Ergebnisse demonstriert werden. Pseudarthrosen traten nur bei 2,6\% der Patienten auf [12]. Kleinere Fehlstellungen in Achse, Länge und Rotation, so die Schlussfolgerung, können am Humerusschaft besser toleriert werden als bspw. an der unteren Extremität. Neuere Studien sehen die konservative Therapie jedoch zunehmend kritisch. Beispielsweise konnte eine Arbeit von Li et al. zeigen, dass ein Rotationsfehler des proximalen Fragmentes von über $20^{\circ}$ nach innen zu einer deutlich verschlechterten Schulterfunktion führt [13]. In einer weiteren Serie von Devers et al. berichteten $75 \%$ der Patienten über eine auffallende kosmetische Fehlstellung, während $25 \%$ das Ergebnis auch funktionell störend empfanden [14]. Eine aktuelle multizentrische Studie aus den USA konnte eine Konversionsrate von $29 \%$ von konservativer zu operativer Therapie feststellen. Hauptursachen waren eine ausbleibende Frakturheilung (60\%), störende Fehlstellungen (24\%) und mangelnde Compliance bez. der Ruhigstellung im Brace [15].

\section{Operative Verfahren}

Die Osteosyntheseverfahren am Humerusschaft können in intramedulläre Nagelung (ante- und retrograd) und extramedulläre Plattenosteosynthese (konventionell, Doppelplatte [DPO] oder minimalinvasiv [MIPO]) aufgeteilt werden. Der Fixateur externe bleibt meist auf die Damage-Control-Phase beschränkt und wird nur selten zur definitiven Ausbehandlung eingesetzt. Im Folgenden werden die technischen Details der einzelnen Verfahren anhand von Fällen fehlgeschlagener Osteosynthesen erläutert und Lösungsmöglichkeiten diskutiert.

\section{Intramedulläre Marknagelosteo- synthese - Frühversagen}

Ob eine Humerusschaftfraktur mittels Marknagelosteosynthese versorgt werden kann, hängt vor allem von der Frakturmorphologie ab. Für eine stabile Verriegelung des Nagels muss der Humeruskopf proximal des Collum chirurgicum intakt sein und die Fraktur sollte distal mindestens $5 \mathrm{~cm}$ von der Fossa olecrani entfernt liegen. Da sich der Markraum distal verjüngt, kann das Nagelende maximal 2,5 cm proximal der Fossa olecrani platziert werden. Für die korrekte Implantationstechnik sei auf die gängigen Lehrbücher und Anleitungen der verschiedenen Hersteller verwiesen. Komplikationen nach Marknagelosteosynthese sind häufig. Eine Arbeit von Baltov et al. konnte in einer Serie mit 111 Patienten eine intraoperative Rate von $47 \%$ chirurgisch-technischer Fehler feststellen. Die häufigsten waren: Überlange proximale Verriegelungsbolzen $(8,1 \%)$, Überstehen des Nagels über Kalottenniveau $(7,2 \%)$ und iatrogene diaphysäre Fraktur (6,3\%). Bei 16,2\% der Patienten war eine Revisionsoperation aufgrund einer Komplikation im Bereich des Schultergelenks notwendig [16]. 


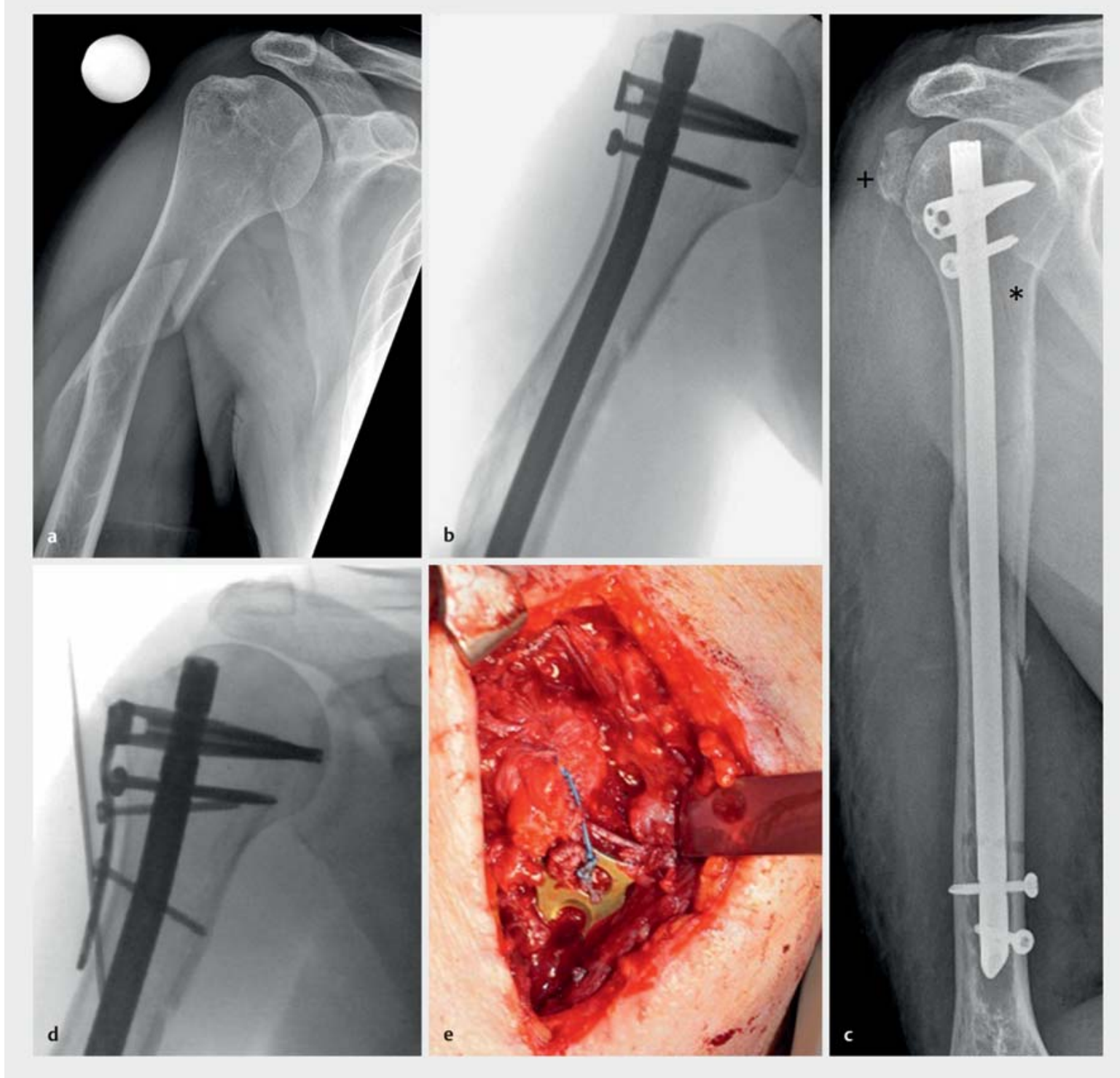

- Abb. 1 Fall eines 51-jährigen, männlichen Patienten. a Initiale Fraktursituation (AO 12A1). b Intraoperatives Bild nach anatomischer Reposition und Verriegelung mit Klinge. c Postoperatives Röntgenbild mit proximalem Frakturausläufer $\left({ }^{*}\right)$ und sekundärer Dislokation des Tuberculum majus (+). d, e Revisionsoperation mit 3,5-mm-T-Platte und Refixation der SSP-Sehne mit Faden-Cerclagen.

\section{Sekundäre Dislokation des Tuberculum majus}

Kritisch für die Wahl des korrekten Osteosyntheseverfahrens präoperativ ist zunächst die adäquate Einschätzung der Frakturmorphologie. A Abb. 1 zeigt den Fall eines 51-jährigen, männlichen Patienten nach ebenerdigem häuslichem Sturz mit einer diaphysären Spiralfraktur (AO 12A1). Der Eintrittspunkt des Nagels ist korrekt gewählt und die Fraktur anatomisch reponiert. Weiterhin wurde für die proximale Verriegelung die Klinge gewählt. Im kurzfristigen postoperativen Verlauf kam es dann zu einer sekundären Dislokation des Tuberculum majus. Kritisch anzumerken ist, dass im postoperativen Röntgen ein weit nach proximal ziehender Frakturausläufer zu sehen ist, der am ehesten bereits seit dem Unfall bestand. Hier bietet der Nagel im Vergleich zur langen Plattenosteosynthese über einen anterolateralen Zugang nur limitierte Möglichkeiten zur Refixation eines proximalen Frakturfragmentes. Bei Fraktur des Tuberculum majus sollte weiterhin unbedingt eine Refixation der SSP-Sehne (SSP: Supraspinatus) an die entsprechend hierfür vorgesehenen Löcher der Verriegelungsklinge erfolgen. In der folgenden Revisionsoperation wurde das proximale Frakturfragment mittels 3,5-mm-T-Platte gefasst und die SSP-Sehne mit Cerclagen an die Platte refixiert. Zur Nachbehandlung wurde ein modifiziertes Schema mit 6 Wochen Ru- 

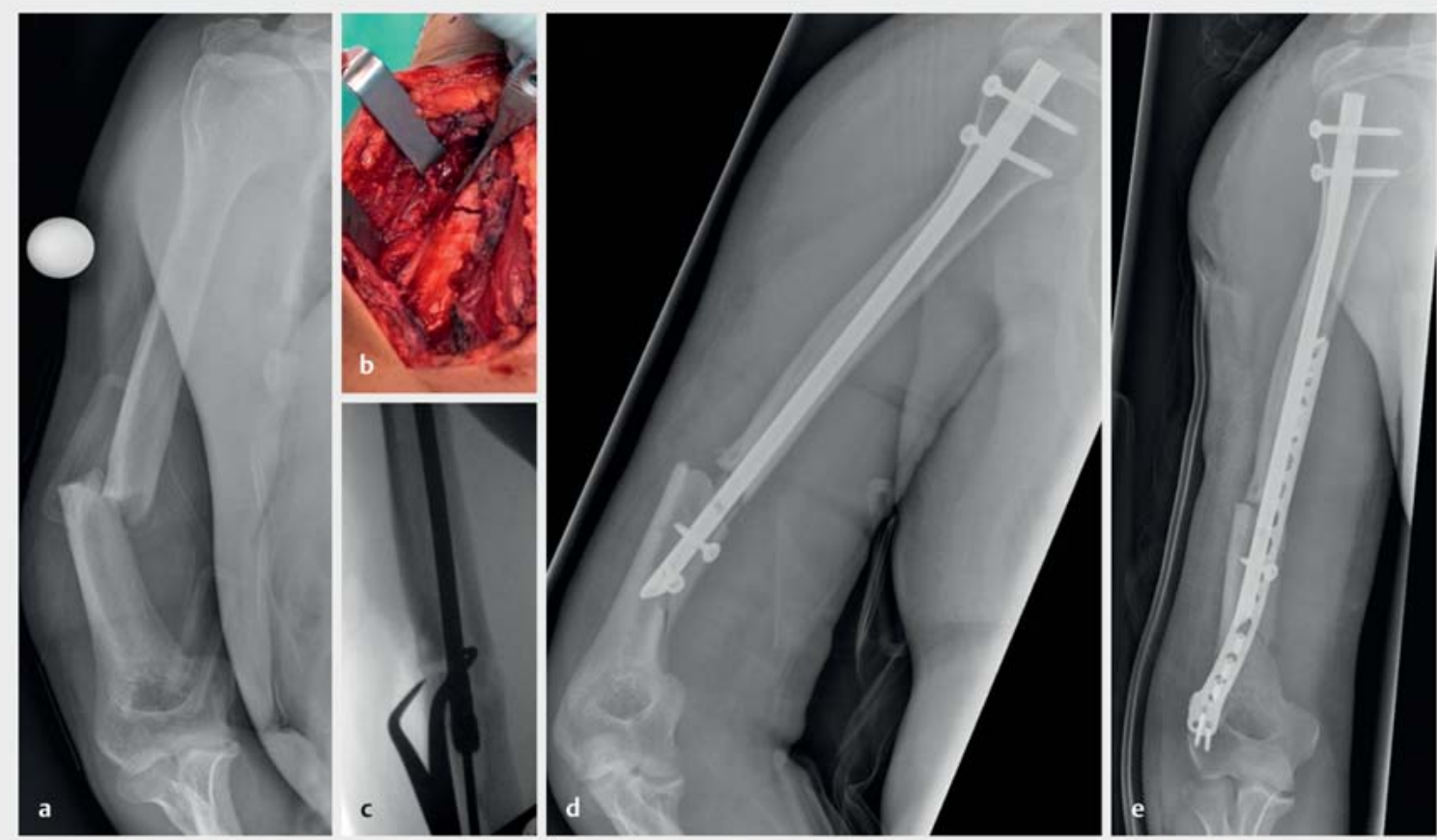

- Abb. 2 Fall eines 46-jährigen Patienten mit Osteogenesis imperfecta (OI). a Initiale Fraktursituation (AO 12A3). b,c Intraoperative Bilder mit Neurolyse des N. radialis, offener Reposition und Aufbohren des Markraumes. $\mathbf{d}$ Periimplantäre Fraktur im distalen Fragment. e Postoperatives Röntgenbild nach Revisionsoperation mit additiver radialer LCP.

higstellung des Oberarms im Gilchrist sowie nur passiver Beübung der Schulter gewählt.

\section{Periimplantäre Fraktur}

- Abb. 2 zeigte den Fall eines 46-jährigen, männlichen Patienten mit Osteogenesis imperfecta (OI). Nach Sturz aus dem Rollstuhl hatte dieser eine Humerusschaftfraktur (AO 12A3) mit begleitender sensomotorischer Radialisparese erlitten. Es wurde ein Nagel der Größe $240 \times 7 \mathrm{~mm}$ implantiert. Der N. radialis wurde über einen anterolateralen Zugang neurolysiert und zeigte sich zwar kontusioniert, aber intakt. Im kurzfristigen postoperativen Verlauf zeigte sich dann eine periimplantäre Fraktur im distalen Fragment. In der Revisionsoperation wurde dann zunächst über einen dorsalen Trizeps-Split-Zugang der N. radialis weiter neurolysiert und die periimplantäre Fraktur mittels Fibertape-Cerclage reponiert. Die Verriegelungsbolzen waren stabil, sodass diese belassenen werden konnten. Anschließend wurde die Fraktur mit einer radialen 3,5-mm-LCP (LCP: Locking Compression Plate) überbrückt. Die Nachbehandlung erfolgte konservativ, mit nur passiver Beübung für 6 Wochen. Die Radialisparese hatte sich in einem Zeitraum von 3 Monaten postoperativ bereits wieder vollständig erholt.

In diesem Fall ist die periimplantäre Fraktur im distalen Fragment mit hoher Wahrscheinlichkeit auf die Grunderkrankung zurückzuführen. Die Knochenqualität ist bei
Ol deutlich reduziert und die Patienten haben häufig multiple Frakturen und eine hohe Pseudarthroserate [17]. Die Eröffnung des Markraumes sowie das Aufbohren gestaltet sich dennoch häufig schwierig. Im vorliegenden Fall wurde über die Fraktur das distale Fragment aufgebohrt. Kritisch anzumerken ist, dass der Nagel gerade die geforderte Mindestlänge zur Verriegelung im distalen Frakturfragment von $5 \mathrm{~cm}$ erreicht.

\section{Intramedulläre Marknagelosteo- synthese - Spätversagen}

Häufigste Ursache für das späte Versagen einer Nagelosteosynthese ist die Pseudarthrose mit nachfolgender Auslockerung des Implantates. Für die operative Behandlung insgesamt (Nagel und Platte) werden in der Literatur Pseudarthroseraten von 2-23\% angegeben. Das Risiko scheint bei Versorgung mit Marknagel etwas höher zu sein. Als Ursache wird die geringere biomechanische Stabilität unaufgebohrter Nägel gesehen [18-21]. Im europäischen Gebiet spricht man bei ausbleibender Konsolidierung der Fraktur bereits nach 4-6 Monaten von einer Pseudarthrose. In den USA ist das Zeitfenster mit 9 Monaten (3 Monate ohne Progress der Frakturheilung) etwas weiter gefasst. Nach den Kriterien der Arbeitsgemeinschaft für Osteosynthesefragen (AO) liegt eine Pseud- 


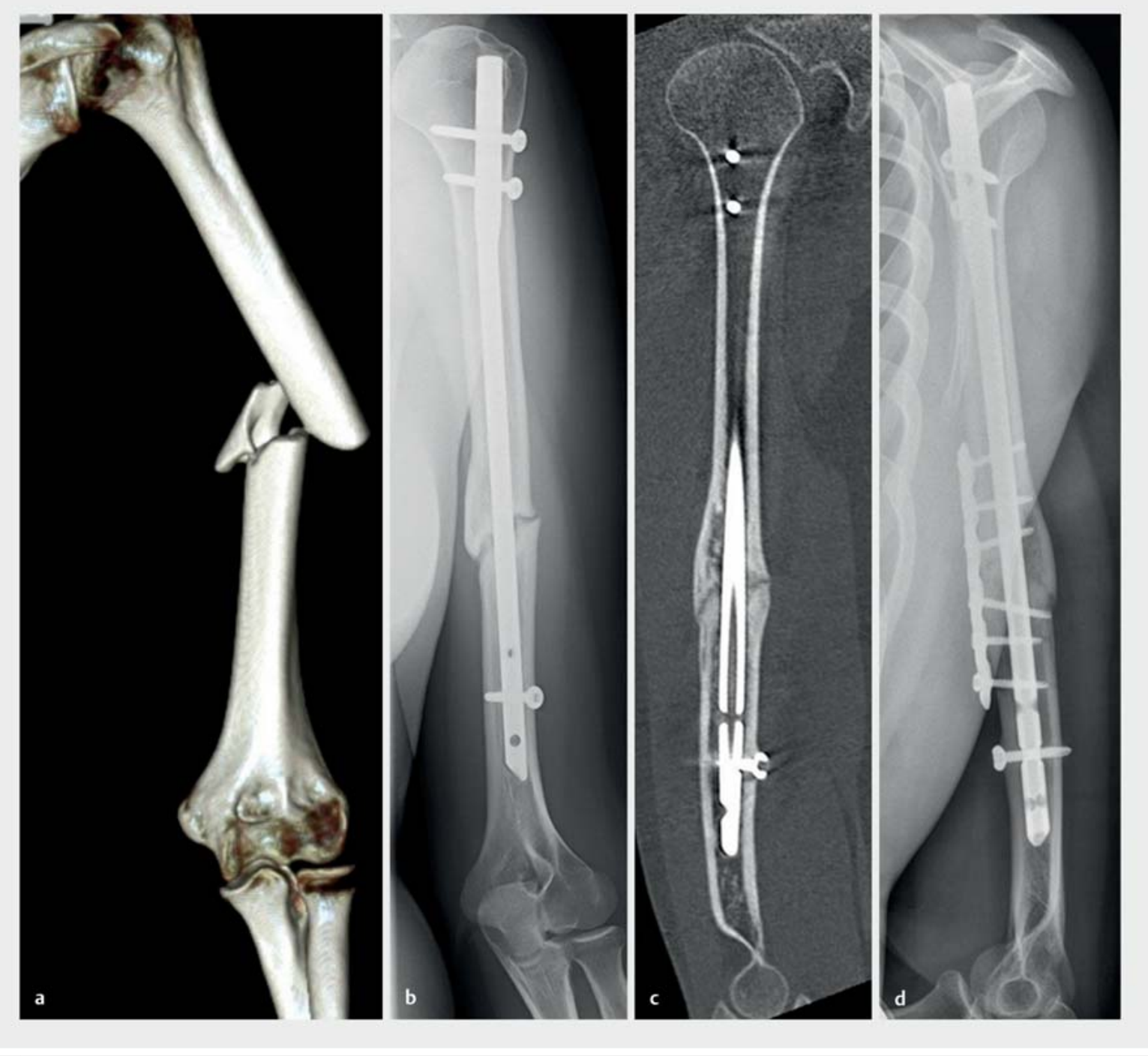

A Abb. 3 Fall einer 23-jährigen Patientin nach Motorradunfall. a Initiale Fraktursituation (AO 12B2). b,c Röntgenbild und CT nach 1,5 Jahren mit Pseudarthrose bei stabilem Implantat. $\mathbf{d}$ Postoperatives Röntgenbild nach additiver ventraler LCP und autologer Spongiosaplastik.

arthrose vor, wenn der Chirurg nicht mehr von einer Heilung ohne erneute operative Intervention ausgeht.

\section{Stabiles Implantat}

Wird eine Pseudarthrose festgestellt, muss zunächst die biologische Aktivität sowie die Stabilität des Marknagels beurteilt werden. Ist das Implantat stabil bei erhaltener biologischer Aktivität, kann die Fraktur in den meisten Fällen durch Erhöhung der Stabilität mittels additiver Plattenosteosynthese über einen anterolateralen Zugang zur Ausheilung gebracht werden. In $>$ Abb. $\mathbf{3}$ ist der Fall einer 23-jährigen Patientin nach Motorradunfall mit diaphysärer Humerusschaftfraktur (AO 12B2) dargestellt. Die initiale osteosynthetische Versorgung erfolgte mittels intramedullärer Marknagelosteosynthese $(240 \times 7 \mathrm{~mm})$. Nach 1,5 Jahren stellte sich die Patientin mit persistierenden, insbesondere belastungsabhängigen Schmerzen und nativradiologisch ausbleibender Konsolidierung bei regelrecht einliegendem Nagel vor. Wir führten eine Revisionsoperation über einen anterolateralen Zugang mit Dekortikation nach Judet, additiver ventraler $3,5-\mathrm{mm}$ LCP und lokaler Spongiosaplastik durch. Um zusätzliche Kompression auf den Frakturspalt zu applizieren, ist hier auch eine Entfernung der distalen Verriegelungsbolzen und exzentrische Bohrung der Plattenlöcher möglich [22]. Zum Ausschluss eines Infektes wurde ebenfalls eine mikrobiologische Probe entnommen.

In einer Serie mit 37 Patienten von Gessmann et al. konnte durch additive ventrale Plattenosteosynthese bei einliegendem Nagel eine Ausheilungsrate von 97\% mit der beschriebenen Technik erreicht werden [23]. Bei atropher Pseudarthrose kann dieses Verfahren natürlich auch 

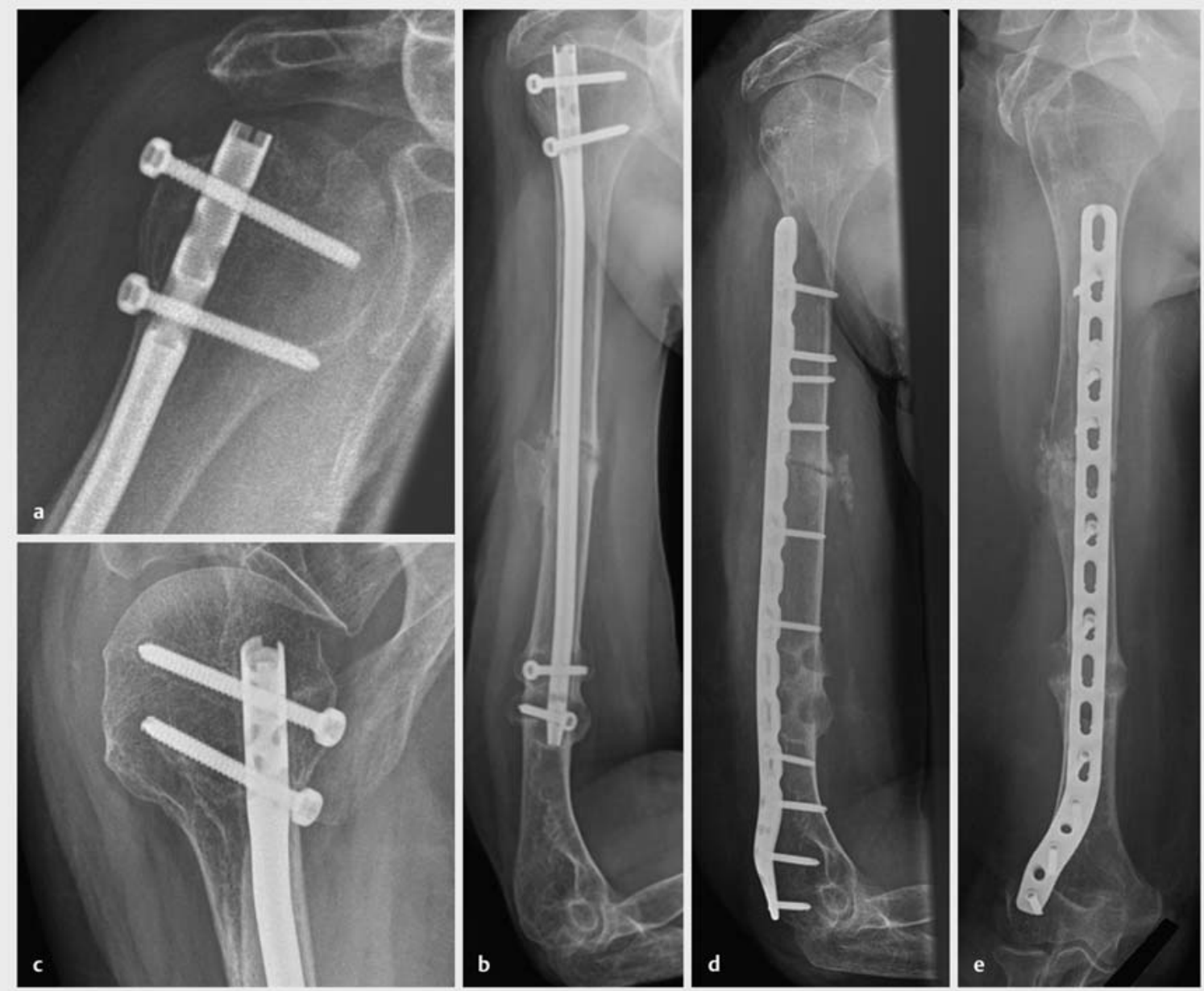

- Abb. 4 Fall einer 65-jährigen Patientin. a-c Röntgenbilder 1 Jahr nach initialer Versorgung mit gelockertem Implantat mit Überstand des Nagels über Kalottenniveau und Pseudarthrose. d, e Operative Versorgung mit autologer Spongiosaplastik und DHP (Fa. Synthes).

mit einer autologen Spongiosaplastik, bspw. vom Beckenkamm, kombiniert werden.

\section{Gelockertes Implantat}

Revisionen bei gelockertem Marknagel sind deutlich aufwendiger und i.d.R. mit einem Verfahrenswechsel auf eine Plattenosteosynthese sowie einer Spongiosaplastik verbunden. In $>$ Abb. 4 ist der Fall einer 65-jährigen Patientin 1 Jahr nach Humerusschaftfraktur dargestellt. Der eingebrachte Nagel ist proximal und distal vollständig gelockert, der Nagel oberhalb der Kalotte nach proximal ausgewandert. Im Bereich des ehemaligen Frakturspaltes liegt eine atrophe Pseudarthrose vor. Präoperativ klagt die Patientin über belastungsabhängige Schmerzen im Oberarm, weiterhin drehe sich der Arm teilweise unkontrolliert bei Belastung. Bei der Revisionsoperation wurde der einliegende Nagel zunächst entfernt und die Patienten dann in Bauchlage gebracht. Hiernach erfolgte die aufwendige Darstellung der Pseudarthrose über einen dorsalen transtrizipitalen Zugang mit langstreckiger Neurolyse des N. radialis. Nach Dekortikation wurde autologe Spongiosa vom Beckenkamm angelagert und eine Reosteosynthese mittels DHP (distale Humerusplatte; Fa. Synthes) durchgeführt. Postoperativ bot die Patienten das Bild einer motorischen Radialisparese, die sich in der weiteren Nachsorge nur sehr langsam regredient zeigte (s. Abschnitt „N. radialis“).

In der aktuellen Literatur werden die besten Ausheilungsergebnisse nach einer Kombination aus Plattenosteosynthese und autologer Spongiosaplastik beschrieben. In einem systematischen Review mit insgesamt 36 Studien konnte eine Ausheilungsrate von 98\% (vs. Revision mit Nagel 88\%) mit der beschriebenen Technik erzielt werden [24]. Im eigenen Vorgehen spielt die Nagelosteosynthese bei Revisionen keine Rolle. 


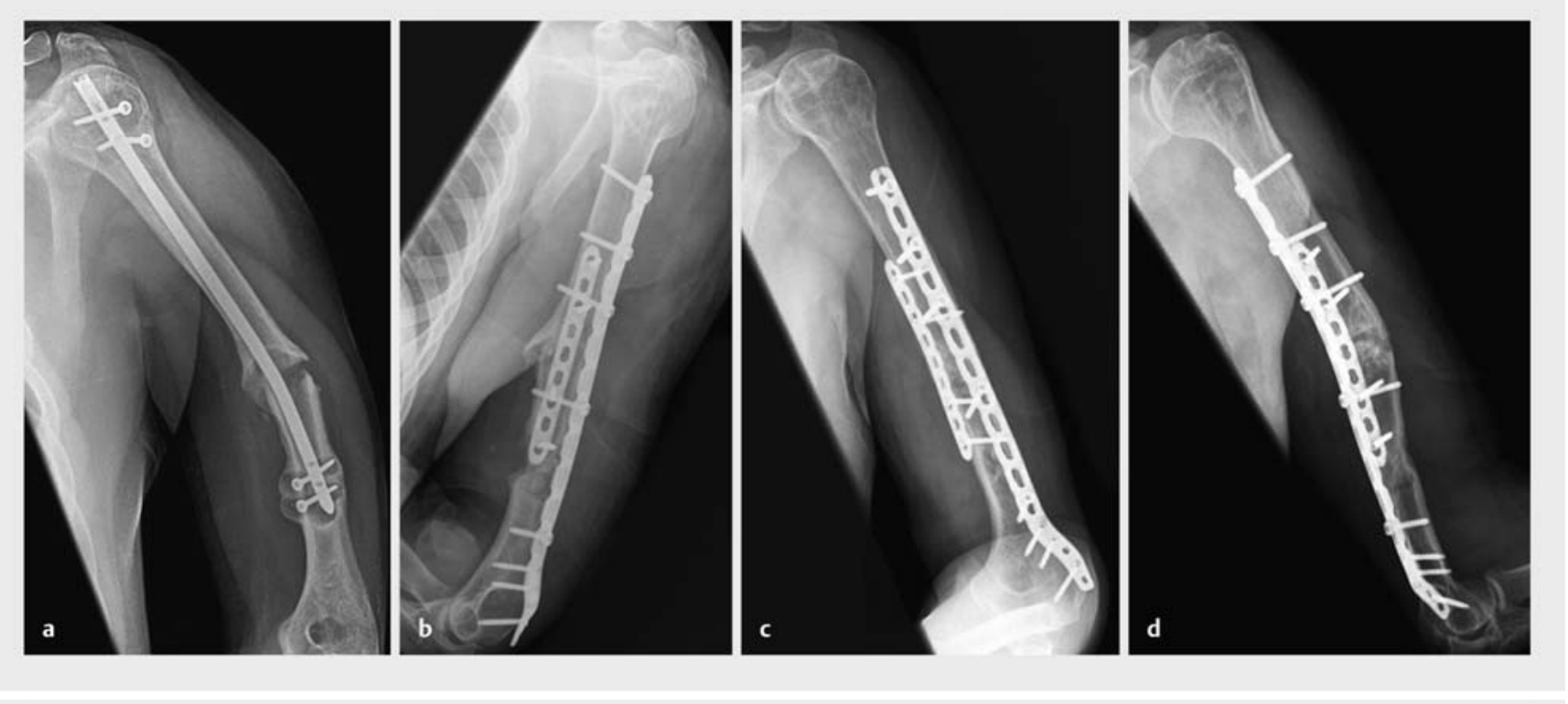

- Abb. 5 Röntgenbilder eines 39-jährigen Patienten. a Auslockerung des Marknagels 1 Jahr nach initialer Versorgung. Revisionsoperation mit Doppelplattenosteosynthese DHP (Fa. Synthes) radial und additiver 3,5-mm-LCP ulnar, zusätzlich autologe Spongiosaplastik. b Seitliches Röntgen. c Anteroposteriores Röntgen. d Situation 1 Jahr postoperativ mit nahezu vollständiger Konsolidierung. Verbiegung der einliegenden Platten mit Achsabweichung (ca. $15^{\circ}$ ) nach erneutem Sturzereignis 6 Monate nach Revision.

Liegt ein Stabilitätsproblem vor, so gibt es zunehmend Evidenz für die Doppelplattenosteosynthese $[25,26]$. In - Abb. 5 ist der Fall eines 39-jährigen Patienten mit Pseudarthrose nach Marknagelosteosynthese dargestellt. Der einliegende Nagel ist 1 Jahr nach der initialen Versorgung vollständig gelockert, die Fraktur nicht konsolidiert. Der Patient hat belastungsabhängige Beschwerden und ist nicht arbeitsfähig. In der Revisionsoperation wird der einliegende Nagel zunächst entfernt und der Markraum zur Anfrischung der Pseudarthrose überbohrt. AnschlieBend erfolgt die Umlagerung des Patienten in Bauchlage und die Resektion der Pseudarthrose über einen dorsalen transtrizipitalen Zugang mit langstreckiger Neurolyse des $\mathrm{N}$. radialis. Hiernach erfolgt eine autologe Spongiosaplastik und Doppelplattenosteosynthese mit einer DHP radial und einer 3,5-mm-LCP ulnar. Die Radialisfunktion war bei dem Patienten postoperativ normal. Bereits 3 Monate nach der Revisionsoperation hatte der Patient keine Beschwerden mehr, konnte seine Arbeit wieder aufnehmen und den Arm voll belasten. Nach 6 Monaten stürzte der Patient erneut, die Röntgenbilder zeigten eine Verbiegung der Platten sowie eine Achsabweichung von ca. $15^{\circ}$. Da sich kein Hinweis auf eine Lockerung der Implantate und der Patient nahezu keine Beschwerden zeigte, wurde die Situation so belassen. Ein Jahr nach Revisionsoperation konnte eine nahezu vollständige Konsolidierung nativradiologisch gesehen werden.

\section{Plattenosteosynthese - Frühversagen}

Grundsätzlich können alle Humerusschaftfrakturen mittels Plattenosteosynthese suffizient versorgt werden. Je nach Frakturlokalisation wird bei eher proximal gelegenen Frakturen (und Frakturausläufern in Richtung Kalotte) ein deltoideopektoraler Zugang mit Verlängerung nach distal entlang des Schaftes im Sinne eines anterolateralen Zugangs gewählt. Frakturen der mittleren und distalen Diaphyse können in Bauchlage über einen dorsalen Zugang mit Trizeps-Split nach sorgsamer Neurolyse des $\mathrm{N}$. radialis versorgt werden. In der Literatur findet sich jedoch, insbesondere bei den dorsalen Zugängen, im Vergleich zur Marknagelosteosynthese eine erhöhte Rate an Radialisläsionen (7,4 vs. 2,6\%) [18]. Eine weitere Technik ist die minimalinvasive Plattenosteosynthese (MIPO) mit eingeschobener langer winkelstabiler Platte über mehrere anteriore/anterolaterale Zugänge, bei der auf eine Darstellung der Fraktur und anatomisch exakte Reposition verzichtet wird. Erste Studien zeigen hier vergleichbare Ergebnisse zu den etablierten Verfahren [27, 28].

\section{Repositionsverlust}

Frühversagen nach Plattenosteosynthese tritt meist aufgrund unzureichender Stabilität mit nachfolgendem Repositionsverlust auf. In > Abb. $\mathbf{6}$ ist der Fall eines 46 -jährigen männlichen Patienten mit Humerusschaftfraktur (AO 12B2) nach Pkw-Unfall dargestellt. Nach initialer Osteosynthese über einen dorsalen Trizeps-Split-Zugang stellte sich der Patient 6 Wochen postoperativ mit einem sekun- 

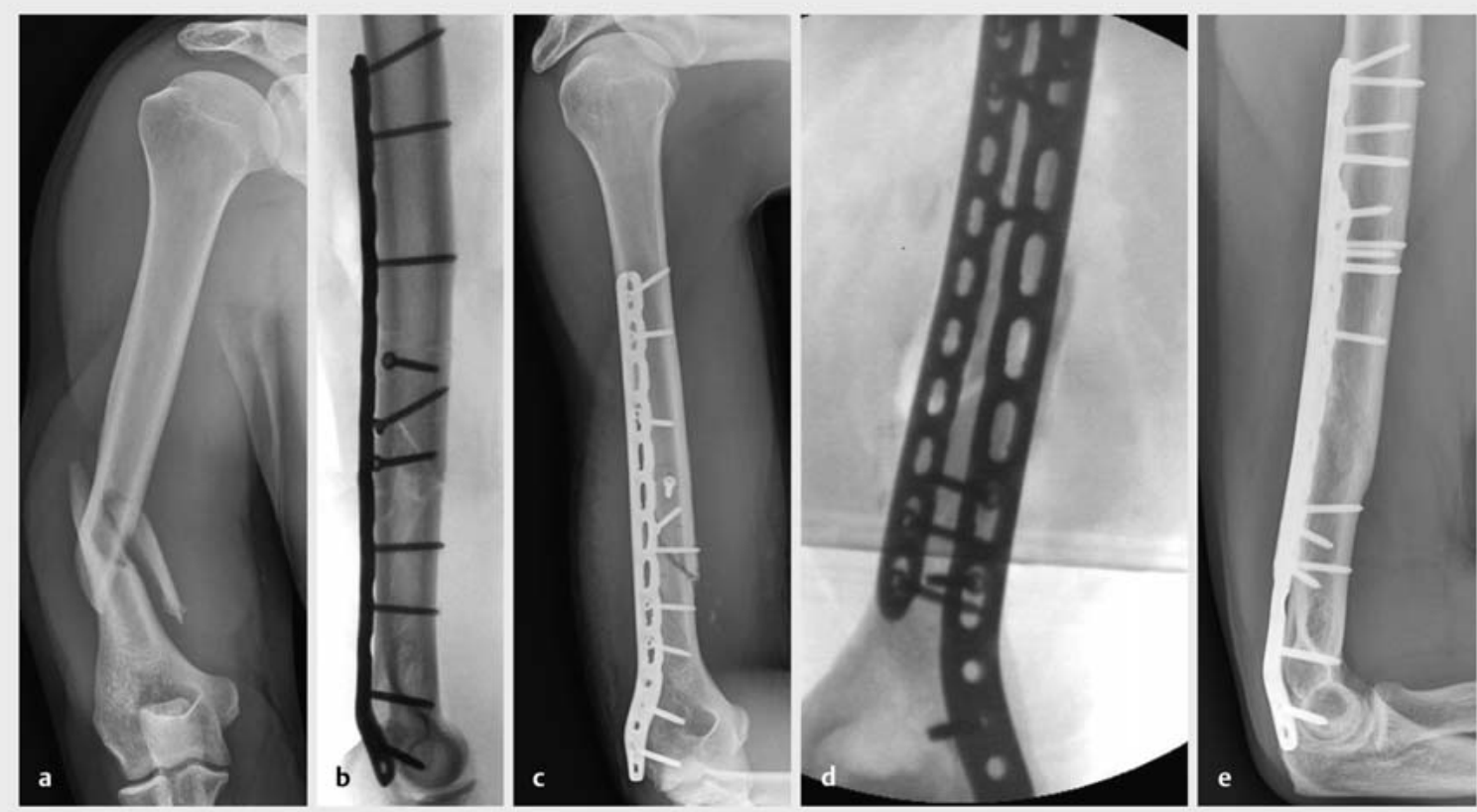

- Abb. 6 Fall eines 46-jährigen Patienten. a Initiale Fraktursituation (AO 12B2). b Intraoperative Röntgenbilder nach Osteosynthese mittels DHP (Fa. Synthes). c Postoperatives Röntgenbild mit sekundärer Dislokation der Fraktur. $\mathbf{d}$ Operative Revision mit additiver 3,5-mm-LCP ulnar. e Konsolidierung der Fraktur 1 Jahr postoperativ.

dären Repositionsverlust erneut vor. Aufgrund der vermuteten Instabilität und daraus folgender Gefahr der Pseudarthrosenbildung wurde eine Revisionsoperation mit additiver ulnarer 3,5-mm-LCP durchgeführt. Hierunter konnte die Fraktur dann problemlos zur Ausheilung gebracht werden.

\section{N. radialis}

Die enge anatomische Lagebeziehung des N. radialis zum Humerusschaft verursacht bei Frakturen nicht selten Ausfallerscheinungen im autonomen Versorgunggebiet. Hierzu zählen motorisch die Handgelenksextensoren, bei deren Ausfall es zum klassischen Bild der „Fallhand“ kommt. Sensorisch sind vor allem Anteile der Dorsalseite des D I-D III betroffen. Als sensorisches Autonomgebiet gilt ein kleiner Bereich interdigital zwischen D I und D II.

Eine Metaanalyse von Shao et al. konnte zeigen, dass eine Radialisparese in $11,8 \%$ der Humerusschaftfrakturen auftritt (532 Paresen bei 4517 erfassten Frakturen). Dabei waren vor allem Frakturen des mittleren und mittleren bis distalen Schaftdrittels mit einem erhöhten Risiko für eine Parese assoziiert. In der Studie wurden von den 532 Patienten mit Parese insgesamt 397 (75\%) operativ exploriert. Hiervon zeigten 184 Patienten (46\%) einen intakten oder kontusionierten Radialis. Bei 53 Patienten (13\%) war der Nerv im Frakturspalt eingeklemmt. Bei insgesamt 93 Patienten (23\%) zeigte sich eine vollständige Kontinuitätsunterbrechung und/oder wurde ein Nerven- transplantat verwendet. Insgesamt zeigten 87,9\% der Patienten, bei denen frühzeitig eine operative Exploration des Nerven durchgeführt wurde, eine Erholung der Funktion. Bei verzögerter Exploration (> 8 Wochen) konnte eine Rate von 65,8\% festgestellt werden. Die spontane Erholungsrate ohne operative Exploration lag bei 70,7\%. Der Unterschied zwischen den Gruppen war statistisch nicht signifikant. Im Mittel zeigte sich eine Erholung der Radialisfunktion nach 6,1 Monaten [29].

Insbesondere bei Frakturen, die gut mittels Marknagelosteosynthese versorgt werden könnten, stellt sich regelhaft die Frage nach einer operativen Exploration des N. radialis. Der hohen Rate an Spontanremissionen steht eine nicht zu unterschätzende Rate an chirurgisch behebbaren Komplikationen (Einklemmung des Nerven im Frakturspalt bis zur kompletten Kontinuitätsunterbrechung) gegenüber. Im Einzelfall bleibt deswegen die chirurgische Nervenexploration gerechtfertigt.

\section{Plattenosteosynthese - Spätversagen}

Die Rate an Pseudarthrosen ist, wie oben dargestellt, im Vergleich zur Marknagelosteosynthese bei Plattenosteosynthese etwas geringer und wird in neueren Studien mit ungefähr $10 \%$ angegeben [30]. In $>$ Abb. 7 ist der Fall einer 75-jährigen Patientin gezeigt, die sich 2 Jahre nach initialer Versorgung mit Schmerzen und Instabilität vorstellte. Die nativradiologische Bildgebung zeigte einen 

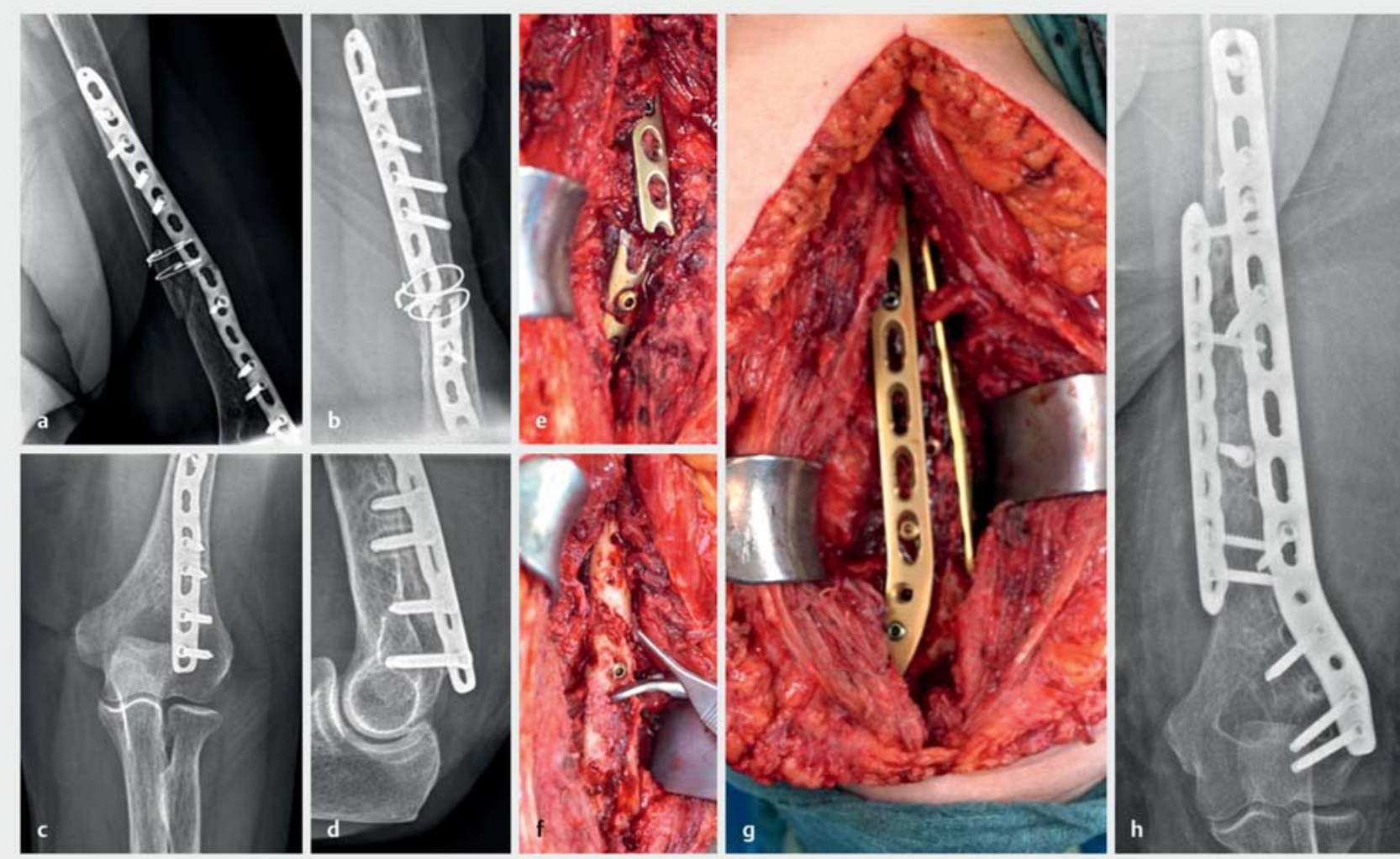

Abb. 7 Fall einer 75-jährigen Patientin. a-d Röntgenbilder 2 Jahre nach initialer Versorgung mit Plattenbruch und Auslockerung der distalen Schrauben. e-g Intraoperative Bilder der gebrochenen Platte, Spongiosaplastik (f) und Doppelplattenosteosynthese. h Postoperatives Röntgenbild.

Bruch der einliegenden 3,5-mm-Platte sowie eine atrophe Pseudarthrose. In der dann durchgeführten Revisionsoperation wurden die Platte sowie die einliegenden Cerclagen zunächst entfernt. Die distalen Schrauben waren vollständig gelockert. Es erfolgte eine Dekortikation sowie lokales Aufbohren des Schaftes mit dem 4,5-mmBohrer. Hiernach wurde eine lokale autologe Spongiosaplastik durchgeführt. Anschließend erfolgte die Reosteosynthese mit DHP (Fa. Synthes) radial sowie additiver ulnarer 3,5-mm-LCP.

Als kritisch in diesem Fall ist zum einen die initiale Versorgung mittels alleiniger 3,5-mm-Platte zu bewerten. Hier konnte keine ausreichende Stabilität erreicht werden, was letztlich zu einer Auslockerung des Implantates und Pseudarthrose geführt hat. Weiterhin liegen die beiden eingebrachten Cerclagen sehr frakturnah und zusätzlich eng zusammen. Dies hat möglichweise zu einer verschlechterten Durchblutungssituation geführt.

Mit der beschriebenen Technik (Plattenosteosynthese und autologe Spongiosaplastik) kann die Mehrzahl der Pseudarthrosen zur Ausheilung gebracht werden. In einer Serie mit 125 Humerusschaftpseudarthrosen benötigten jedoch $16 \%$ der Patienten eine zusätzliche Operation nach Revision, bis eine Konsolidierung festgestellt werden konnte [30]. In diesen Fällen ist die Doppelplattenosteosynthese ein geeignetes Verfahren, um zusätzliche Stabilität zu erreichen.

\section{Infekt}

Infekte nach Humerusschaftfraktur sind insgesamt eher selten. In der Literatur sind Raten von $4 \%$ nach Plattenosteosynthese und 1,6\% nach Marknagelosteosynthese angegeben [31]. In > Abb. 8 ist der Fall einer 44-jährigen Patientin dargestellt, die sich 2 Jahre nach initialer Versorgung einer offenen distalen Humerusschaftfraktur vorstellte. Klinisch bestand ein sezernierender Fistelgang sowie laborchemisch moderat erhöhte Entzündungsparameter (CRP 2,5 mg/dl). Intraoperativ zeigte sich eine ausgeprägte Infektpseudarthrose mit Beteiligung nahezu des gesamten distalen Markraumes sowie ausgelockerten Implantaten. In den mikrobiologischen Proben wurde ein Staphylococcus aureus festgestellt. In mehreren Operationen musste letztlich ein großer Teil des distalen diaphysären Schaftanteils reseziert werden. Zur externen Stabilisierung wurde ein Fixateur angelegt sowie als Platzhalter eine Palacos-Plombe eingesetzt. Diese wurde zur zusätzlichen Stabilisierung mittels 2 Kirschner-Drähten 

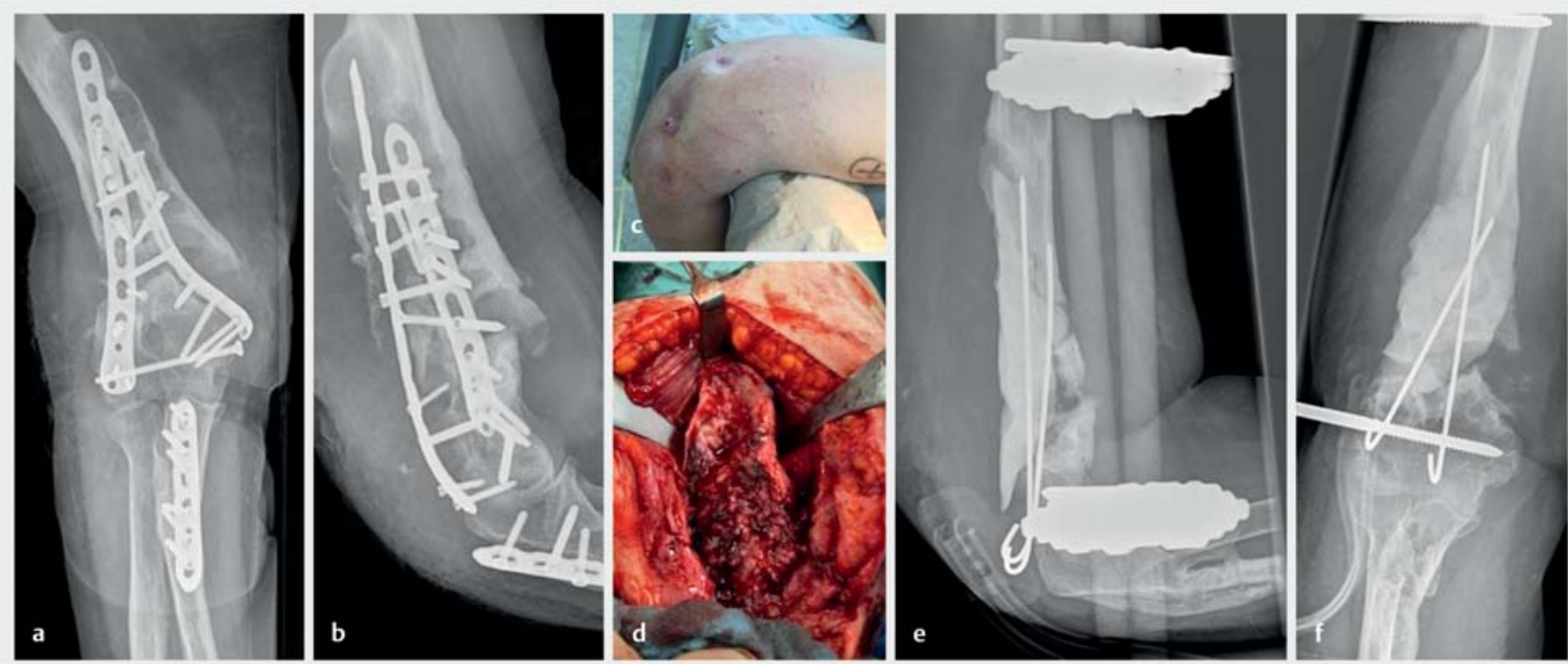

- Abb. 8 Röntgen- und klinische Bilder einer 44-jährigen Patientin. a, b Infektpseudarthrose 2 Jahre nach offener distaler Humerusschaftfraktur. c, $\mathbf{d}$ Intraoperativer Befund mit Beteiligung des gesamten distalen Markraumes. e, $\mathbf{f}$ Postoperative Bilder mit Palacos-Plombe und Anlage eines Fixateur externe.

> Tab. 1 Zusammenfassung der Komplikationen und Lösungsmöglichkeiten.

\begin{tabular}{|c|c|c|c|c|}
\hline & \multicolumn{2}{|l|}{ Marknagel } & \multicolumn{2}{|l|}{ Platte } \\
\hline & Komplikation & Lösung & Komplikation & Lösung \\
\hline $\begin{array}{l}\text { Früh- } \\
\text { versagen }\end{array}$ & $\begin{array}{l}\text { sekundäre Dislokation } \\
\text { Tuberculum majus } \\
\text { periimplantäre Fraktur } \\
\text { weitere: } \\
\text { Überstand des Nagels } \\
\text { überlange Verriege- } \\
\text { lungsbolzen } \\
\text { iatrogene Fraktur } \\
\text { Distraktion im Fraktur- } \\
\text { spalt }\end{array}$ & $\begin{array}{l}\text { Reosteosynthese mit 3,5-mm-T- } \\
\text { Platte, Refixation SSP an Platte und } \\
\text { Verriegelungsklinge } \\
\text { additive Plattenosteosynthese, } \\
\text { Cerclagen, Belassen des Nagels } \\
\text { kürzerer Nagel, neue Verriegelung } \\
\text { weiter distal } \\
\text { Wechsel der Bolzen } \\
\text { Cerclagen } \\
\text { Rückschlagen des Nagels bei nur dis- } \\
\text { taler Verriegelung, Benutzen des } \\
\text { Kompressionsmechanismus proximal }\end{array}$ & $\begin{array}{l}\text { sekundäre Dislokation } \\
\text { Radialisparese }\end{array}$ & $\begin{array}{l}\text { additive ulnare Platte } \\
3,5 \mathrm{~mm} \\
\text { chirurgische Exploration } \\
\text { und Neurolyse, danach } \\
\text { abwartendes Verhalten }\end{array}$ \\
\hline $\begin{array}{l}\text { Spät- } \\
\text { versagen }\end{array}$ & Pseudarthrose & $\begin{array}{l}\text { stabiles Implantat: additive 3,5-mm- } \\
\text { Platte und autologe Spongiosaplastik } \\
\text { gelockertes Implantat: Verfahrens- } \\
\text { wechsel, Dekortikation, Doppel- } \\
\text { plattenosteosynthese und autologe } \\
\text { Spongiosaplastik }\end{array}$ & Pseudarthrose & $\begin{array}{l}\text { Dekortikation, Doppel- } \\
\text { plattenosteosynthese } \\
\text { und autologe Spongiosa- } \\
\text { plastik }\end{array}$ \\
\hline
\end{tabular}


im Knochen fixiert. Antibiotisch wurde die Patientin initial mit Vancomycin und Rifampicin behandelt, nach Erhalt des Antibiogramms konnte auf Flucloxacillin für 6 Wochen gewechselt werden. Zur definitiven Ausversorgung ist eine Auffüllung des Defektes mit Spongiosa in Masquelet-Technik geplant. Hierzu sind in der Literatur für den Humerusschaft lediglich Einzelfallbeschreibungen zu finden [32-34].

\section{Zusammenfassung}

Fehlgeschlagene Osteosynthesen bei Humerusschaftfraktur lassen sich in Früh- und Spätversagen einteilen. Die wichtigsten Komplikationen und operative Lösungsmöglichkeiten sind in > Tab. 1 zusammengefasst.

Prinzipiell lässt sich feststellen, dass bei Marknagelosteosynthesen die adäquate chirurgische Technik bei der Implantation von entscheidender Bedeutung ist, insbesondere, um Komplikationen im Bereich des Schultergelenkes zu vermeiden. Die Pseudarthrosenrate ist im Vergleich zur Plattenosteosynthese etwas erhöht. Bei stabilem Implantat kann eine additive ventrale Platte in den meisten Fällen ausreichend sein, um eine Konsolidierung zu erreichen. Bei gelockertem Implantat lassen sich mit einem Verfahrenswechsel mit Plattenosteosynthese und autologer Spongiosaplastik die meisten Fälle zur Ausheilung bringen.

Die Plattenosteosynthese geht mit einer etwas erhöhten Rate an Radialiskomplikationen einher. Im Einzelfall ist hier eine chirurgische Exploration immer gerechtfertigt. Die Doppelplattenosteosynthese ist insbesondere im Revisionsfall eine gute Möglichkeit, um die Primärstabilität zu erhöhen.

Infekte sind insgesamt selten, es gelten die gleichen Prinzipien der Behandlung wie an anderen Lokalisationen mit Entfernung des einliegenden Fremdmaterials, chirurgischem Débridement, Anlage eines Fixateur externe und antibiotischer Therapie.

\section{Interessenkonflikt}

Die Autorinnen/Autoren geben an, dass kein Interessenkonflikt besteht.

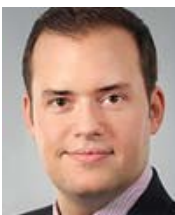

\section{J. Christoph Katthagen}

Priv.-Doz. Dr. med., Oberarzt, Klinik und Poliklinik für Unfall-, Hand- und Wiederherstellungschirurgie, Universitätsklinik Münster

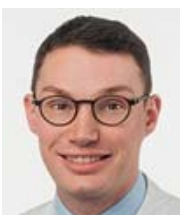

\section{Philipp A. Michel}

Dr. med., Facharzt, Klinik und Poliklinik für Unfall-, Hand- und Wiederherstellungschirurgie, Universitätsklinik Münster

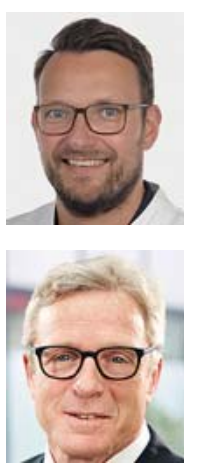

\section{Benedikt Schliemann}

Priv.-Doz. Dr. med., Oberarzt, Klinik und Poliklinik für Unfall-, Hand- und Wiederherstellungschirurgie, Universitätsklinik Münster

\section{Michael J. Raschke}

Univ.-Prof. Dr. med., Direktor der Klinik und Poliklinik für Unfall-, Hand- und Wiederherstellungschirurgie, Universitätsklinik Münster

Korrespondenzadresse

Priv.-Doz. Dr. med. J. Christoph Katthagen

Klinik für Unfall-, Hand- und Wiederherstellungschirurgie Universitätsklinik Münster

Waldeyer Straße 1

48149 Münster

Tel.: 0251/835-9218

Fax: 0251/835-6318

Christoph.Katthagen@ukmuenster.de

\section{Literatur}

[1] Court-Brown CM, Caesar B. Epidemiology of adult fractures: A review. Injury 2006; 37: 691-697. doi:10.1016/j.injury.2006. 04.130

[2] Ekholm R, Adami J, Tidermark J et al. Fractures of the shaft of the humerus. An epidemiological study of 401 fractures. J Bone Joint Surg Br 2006; 88: 1469-1473. doi:10.1302/ 0301-620X.88B11.17634

[3] Kim SH, Szabo RM, Marder RA. Epidemiology of humerus fractures in the United States: nationwide emergency department sample, 2008. Arthritis Care Res (Hoboken) 2012; 64: 407414. doi:10.1002/acr.21563

[4] Ji C, Li J, Zhu Y et al. Assessment of incidence and various demographic risk factors of traumatic humeral shaft fractures in China. Sci Rep 2019; 9: 1965. doi:10.1038/s41598-01838035-y

[5] Mahabier KC, Hartog DD, Van Veldhuizen J et al. Trends in incidence rate, health care consumption, and costs for patients admitted with a humeral fracture in The Netherlands between 1986 and 2012. Injury 2015; 46: 1930-1937. doi:10.1016/j. injury.2015.07.025 
[6] Bergdahl C, Ekholm C, Wennergren D et al. Epidemiology and patho-anatomical pattern of 2,011 humeral fractures: data from the Swedish Fracture Register. BMC Musculoskelet Disord 2016; 17: 159. doi:10.1186/s12891-016-1009-8

[7] Rose SH, Melton LJ 3rd, Morrey BF et al. Epidemiologic features of humeral fractures. Clin Orthop Relat Res 1982; 168: 24-30. doi:10.1097/00003086-198208000-00003

[8] Tytherleigh-Strong G, Walls N, McQueen MM. The epidemiology of humeral shaft fractures. J Bone Joint Surg Br 1998; 80: 249-253. doi:10.1302/0301-620x.80b2.8113

[9] Biber R, Bail H], Gesslein M. [Humeral shaft fractures]. Unfallchirurg 2018; 121: 747-758. doi:10.1007/s00113-018-05334

[10] Stedtfeld HW, Biber R. Proximal third humeral shaft fractures - a fracture entity not fully characterized by conventional AO classification. Injury 2014; 45 (Suppl. 1): S54-S59. doi:10.1016/j.injury.2013.10.030

[11] Ekholm R, Ponzer S, Tornkvist $\mathrm{H}$ et al. The Holstein-Lewis humeral shaft fracture: aspects of radial nerve injury, primary treatment, and outcome. J Orthop Trauma 2008; 22: 693697. doi:10.1097/BOT.0b013e31818915bf

[12] Sarmiento A, Zagorski JB, Zych GA et al. Functional bracing for the treatment of fractures of the humeral diaphysis. J Bone Joint Surg Am 2000; 82: 478-486. doi:10.2106/00004623200004000-00003

[13] Li Y, Wang C, Wang M et al. Postoperative malrotation of humeral shaft fracture after plating compared with intramedullary nailing. J Shoulder Elbow Surg 2011; 20: 947-954. doi:10.1016/j.jse.2010.12.016

[14] Devers BN, Lebus GF, Mir HR. Incidence and functional outcomes of malunion of nonoperatively treated humeral shaft fractures. Am J Orthop (Belle Mead NJ) 2015; 44: E434-E437

[15] Serrano R, Mir HR, Sagi HC et al. Modern results of functional bracing of humeral shaft fractures: a multicenter retrospective analysis. J Orthop Trauma 2020. doi:10.1097/BOT. 0000000000001666

[16] Baltov A, Mihail R, Dian E. Complications after interlocking intramedullary nailing of humeral shaft fractures. Injury 2014; 45 (Suppl. 1): S9-S15. doi:10.1016/j.injury.2013.10.044

[17] Chiarello E, Donati D, Tedesco G et al. Conservative versus surgical treatment of osteogenesis imperfecta: a retrospective analysis of 29 patients. Clin Cases Miner Bone Metab 2012; 9 : 191-194

[18] Gottschalk MB, Carpenter W, Hiza E et al. Humeral shaft fracture fixation: incidence rates and complications as reported by American Board of Orthopaedic Surgery Part II candidates. J Bone Joint Surg Am 2016; 98: e71. doi:10.2106/JBJS.15.01049

[19] Heineman DJ, Bhandari M, Poolman RW. Plate fixation or intramedullary fixation of humeral shaft fractures-an update. Acta Orthop 2012; 83: 317-318. doi:10.3109/17453674.2012. 695677

[20] Heineman DJ, Poolman RW, Nork SE et al. Plate fixation or intramedullary fixation of humeral shaft fractures. Acta Orthop 2010; 81: 216-223. doi:10.3109/17453671003635884

[21] McCormack RG, Brien D, Buckley RE et al. Fixation of fractures of the shaft of the humerus by dynamic compression plate or intramedullary nail. A prospective, randomised trial. J Bone Joint Surg Br 2000; 82: 336-339. doi:10.1302/0301-620x. $82 \mathrm{~b} 3.9675$
[22] Schliemann B, Raschke M], Everding J et al. Humerusschaftpseudarthrosen. Obere Extremität 2020. doi:10.1007/ s11678-019-00553-0

[23] Gessmann J, Königshausen M, Coulibaly MO et al. Anterior augmentation plating of aseptic humeral shaft nonunions after intramedullary nailing. Arch Orthop Trauma Surg 2016; 136: 631-638. doi:10.1007/s00402-016-2418-8

[24] Peters RM, Claessen FM, Doornberg JN et al. Union rate after operative treatment of humeral shaft nonunion - a systematic review. Injury 2015; 46: 2314-2324. doi:10.1016/j.injury. 2015.09.041

[25] Michel PA, Katthagen JC, Raschke MJ et al. Doppelplattenosteosynthese bei proximaler Humerusfraktur. Obere Extremität 2019. doi:10.1007/s11678-019-00539-y

[26] Michel PA, Katthagen JC, Heilmann LF et al. Biomechanics of upper extremity double plating. Z Orthop Unfall 2019. doi:10.1055/a-0862-6334

[27] Kulkarni VS, Kulkarni MS, Kulkarni GS et al. Comparison between antegrade intramedullary nailing (IMN), open reduction plate osteosynthesis (ORPO) and minimally invasive plate osteosynthesis (MIPO) in treatment of humerus diaphyseal fractures. Injury 2017; 48 (Suppl. 2): S8-S13. doi:10.1016/ S0020-1383(17)30487-4

[28] van de Wall BJM, Theus C, Link BC et al. Absolute or relative stability in plate fixation for simple humeral shaft fractures. Injury 2019; 50: 1986-1991. doi:10.1016/j.injury.2019.08.004

[29] Shao YC, Harwood P, Grotz MR et al. Radial nerve palsy associated with fractures of the shaft of the humerus: a systematic review. J Bone Joint Surg $\mathrm{Br}$ 2005; 87: 1647-1652. doi:10.1302/0301-620X.87B12.16132

[30] Wiss DA, Garlich JM. Healing the index humeral shaft nonunion: risk factors for development of a recalcitrant nonunion in 125 patients. J Bone Joint Surg Am 2020; 102: 375-380. doi:10.2106/JBJS.19.01115

[31] Pidhorz L. Acute and chronic humeral shaft fractures in adults. Orthop Traumatol Surg Res 2015; 101 (1 Suppl.): S41-S49. doi:10.1016/j.otsr.2014.07.034

[32] Masquelet AC, Kishi T, Benko PE. Very long-term results of post-traumatic bone defect reconstruction by the induced membrane technique. Orthop Traumatol Surg Res 2019; 105: 159-166. doi:10.1016/j.otsr.2018.11.012

[33] Azi ML, Teixeira AAA, Cotias RB et al. Induced-membrane technique in the management of posttraumatic bone defects. JBJS Essent Surg Tech 2019; 9: e22. doi:10.2106/JBJS.ST.18. 00099

[34] Kombate NK, Walla A, Ayouba G et al. Reconstruction of traumatic bone loss using the induced membrane technique: preliminary results about 11 cases. J Orthop 2017; 14: 489494. doi:10.1016/j.jor.2017.06.009

\section{Bibliografie}

DOI https://doi.org/10.1055/a-1125-6193 online publiziert 28.05.2020 | OP-JOURNAL 2020; 36: 102112 ๑ Georg Thieme Verlag KG Stuttgart · New York ISSN 0178-1715 